\title{
Grain refinement at the nanoscale via mechanical twinning and dislocation interaction in a nickel-based alloy
}

\author{
N.R. Tao \\ Shenyang National Laboratory for Materials Science, Institute of Metal Research, Chinese Academy \\ of Sciences, Shenyang 110016, China \\ X.L. Wu \\ State Key Laboratory of Nonlinear Mechanics, Institute of Mechanism, Chinese Academy of Sciences, \\ Beijing 100080, China \\ M.L. Sui \\ Shenyang National Laboratory for Materials Science, Institute of Metal Research, Chinese Academy \\ of Sciences, Shenyang 110016, China \\ J. $\mathrm{Lu}$ \\ LASMIS, University of Technology of Troyes, 10000 Troyes, France \\ K. $\mathrm{Lu}^{\text {a) }}$ \\ Shenyang National Laboratory for Materials Science, Institute of Metal Research, Chinese Academy \\ of Sciences, Shenyang 110016, China
}

(Received 21 July 2003; accepted 2 February 2004)

A nanostructured surface layer was formed on an Inconel 600 plate by subjecting it to surface mechanical attrition treatment at room temperature. Transmission electron microscopy and high-resolution transmission electron microscopy of the treated surface layer were carried out to reveal the underlying grain refinement mechanism.

Experimental observations showed that the strain-induced nanocrystallization in the current sample occurred via formation of mechanical microtwins and subsequent interaction of the microtwins with dislocations in the surface layer. The development of high-density dislocation arrays inside the twin-matrix lamellae provides precursors for grain boundaries that subdivide the nanometer-thick lamellae into equiaxed, nanometer-sized grains with random orientations.

\section{INTRODUCTION}

Due to increasing interest in nanostructured materials in recent years, various synthesis techniques for nanostructured or ultrafine-grained materials have been developed based on the plastic deformation of coarsegrained polycrystals, such as ball milling, ${ }^{1,2}$ cold rolling, ${ }^{3,4}$ equal-channel angular pressing, ${ }^{5,6}$ high-pressure torsion, ${ }^{7,8}$ and surface mechanical attrition. ${ }^{9,10}$ Therefore, understanding the underlying mechanisms of grain refinement induced by plastic straining is crucial, especially at the nanometer scale. On one hand, understanding the mechanism of strain-induced grain refinement is crucial for development of synthesis techniques that are oriented toward practical applications for engineering materials. On the other hand, the grain refinement process at the nanometer scale is of particular academic interest as the plastic deformation takes place at extreme conditions (very large strains and/or high strain rates).

\footnotetext{
a) Address all correspondence to this author.

e-mail: lu@imr.ac.cn

DOI: $10.1557 / J M R .2004 .0227$
}

Many previous investigations showed that the grain refinement process during plastic deformation originates from dislocation activity in some metals and alloys such as $\mathrm{Fe},{ }^{11} \mathrm{Cu},{ }^{12,13} \mathrm{Ni},{ }^{14,15}$ an Al-based alloy, ${ }^{16}$ and low-carbon steels. ${ }^{17}$ Plastic straining induces generation of a high density of lattice dislocations in original grains. These dislocations arrange themselves into various configurations depending on the crystal structure of the material, such as dense dislocation walls on specific slip planes, dislocation tangles, and dislocation cells. Dislocation interactions lead to formation of subgrain boundaries across which small misorientations are created. Further development of these sub-boundaries with increasing strain results in high-angle grain boundaries that subdivide the original grains.

Differing from these previously reported mechanisms, a grain refinement process via formation of mechanical microtwins and subsequent interaction of the microtwins with strain-induced dislocations is introduced in this work. A recently developed technique, surface mechanical attrition treatment (SMAT), was used to create a gradient variation of the strain and strain rate in the 
deformed material from the treated top surface (both are very large) to deep within matrix (essentially zero). The microstructure was then investigated at different depths to reveal the underlying mechanism of grain refinement.

\section{EXPERIMENTAL}

A concentrated Ni-based solid solution, Inconel 600, was subjected to SMAT to achieve a nanostructured surface layer. Its nominal composition was, in wt $\%, 15.5 \mathrm{Cr}$, $8 \mathrm{Fe}, 0.1 \mathrm{C}, 0.8 \mathrm{Mn}, 0.38 \mathrm{Si}, 0.4 \mathrm{Cu}$, with the balance Ni. The dimensions of the treated plate were $8 \times 60 \times$ $60 \mathrm{~mm}^{3}$, and the treated surface was polished with silicon carbide papers. The grain size of the $\mathrm{Ni}$ solid solution before SMAT was approximately 10-30 $\mu \mathrm{m}$.

The SMAT setup and procedures have been described in detail elsewhere. ${ }^{11,18}$ In this work, a system vibration frequency of $20 \mathrm{kHz}$ and stainless steel shots of $3 \mathrm{~mm}$ in diameter were selected, and the Inconel 600 samples were treated at room temperature for $12 \mathrm{~min}$.

Microstructural features of the surface layer in the SMAT Inconel 600 sample were characterized using transmission electron microscopy (TEM) and highresolution TEM (HRTEM) on a Philips (Holland) EM 420 transmission electron microscope (operated at a voltage of $120 \mathrm{kV}$ ) and a JEOL (Tokyo, Japan) 2010 highresolution transmission electron microscope (at $200 \mathrm{kV}$ ), respectively. Cross-sectional samples for TEM observations were prepared by electroplating a nickel layer of $1.5 \mathrm{~mm}$ thickness on the treated surface. Cross-sectional disks were cut from the electroplated sample using an electrical discharge technique and were then thinned by dimple grinding. Finally, samples for TEM observations were thinned by ion milling at low temperatures.

\section{RESULTS AND DISCUSSION}

\section{A. Dislocation slip in the low-strain region}

In the region of low strain and strain rate, adjacent to the strain-free matrix (depth $>40 \mu \mathrm{m}$ ), TEM observations showed that the plastic deformation mode was dislocation slip. Microstructures in this region are characterized by planar dislocation arrays formed on different $\{111\}$ slip planes, as seen in Fig. 1. The dislocation configurations in the current sample differ substantially from those observed in $\mathrm{Fe}^{11}$ and an Al-based alloy ${ }^{16}$ after SMAT, in which dislocation walls or cell structures developed.

\section{B. Characteristics of mechanical microtwins}

With decreasing depth from the surface (increasing strain and strain rate), the increasing dislocation density results in an increase of the flow stress. The critical stress for twin formation may then be exceeded, and

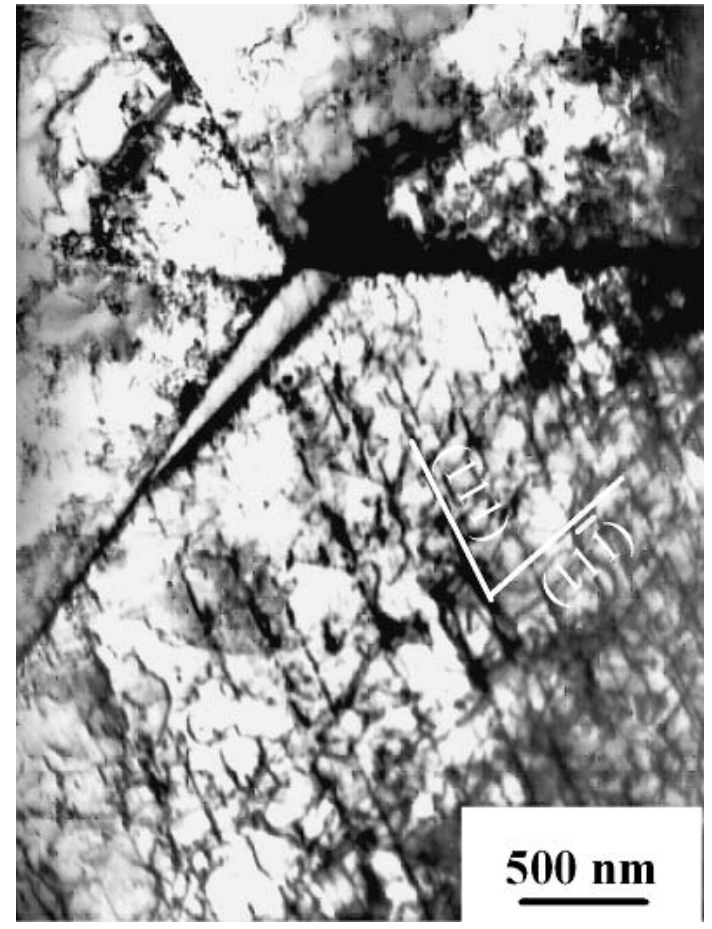

FIG. 1. A cross-sectional TEM image showing the dislocation configurations in the low-strain region.

mechanical twinning becomes the dominant deformation mode. Figure 2 shows a typical observation of the highdensity mechanical microtwins that are of $\{111\}\langle 112\rangle$ type, located approximately $22 \mu \mathrm{m}$ from the top surface. These microtwins are parallel to each other, and their thickness is as small as a few nanometers. The length of these microtwins is several hundred nanometers. The density of microtwins is so high that their thickness is comparable to that of the corresponding lamellar matrix. Formation of a high density of ultrathin (nanometer scale) mechanical twins implies that the surface layer has undergone plastic deformation at a very high strain rate (estimated to be about $10^{3}$ to $10^{4} \mathrm{~s}^{-1}$ ) without significant temperature rise during the SMAT process, so that a high density of deformation-induced dislocations in the matrix suppresses the growth tendency of the mechanical twins in this region. ${ }^{19}$ Apparently, formation of the highdensity microtwins introduced a large amount of twin boundaries that subdivided the original coarse grains into lamellar twin-matrix blocks.

A twin boundary is a particularly simple case of a periodic boundary or a "special" large-angle boundary with a very high degree of coincidence. However, in the SMAT Inconel 600 sample, we found that the orientation angles across twin-matrix interfaces deviate from the expected value of $70.5^{\circ}$ for the $\{111\}\langle 112\rangle$ twin boundary, as indicated in Fig. 2. Spots in the selected-area electrondiffraction (SAED) pattern are elongated. Based on the SAED patterns, the deviation of the orientation angle 


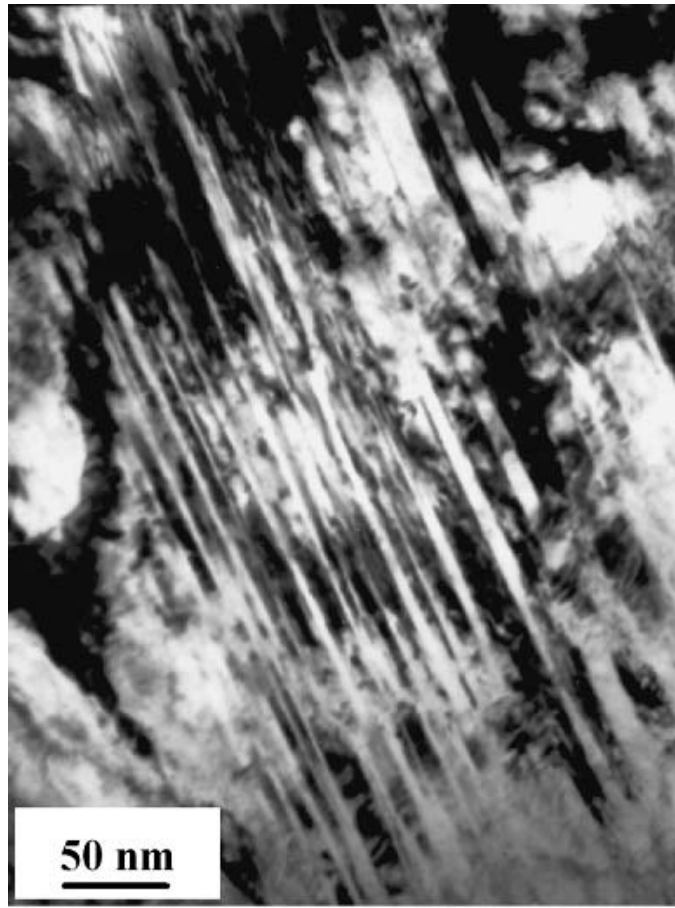

(a)

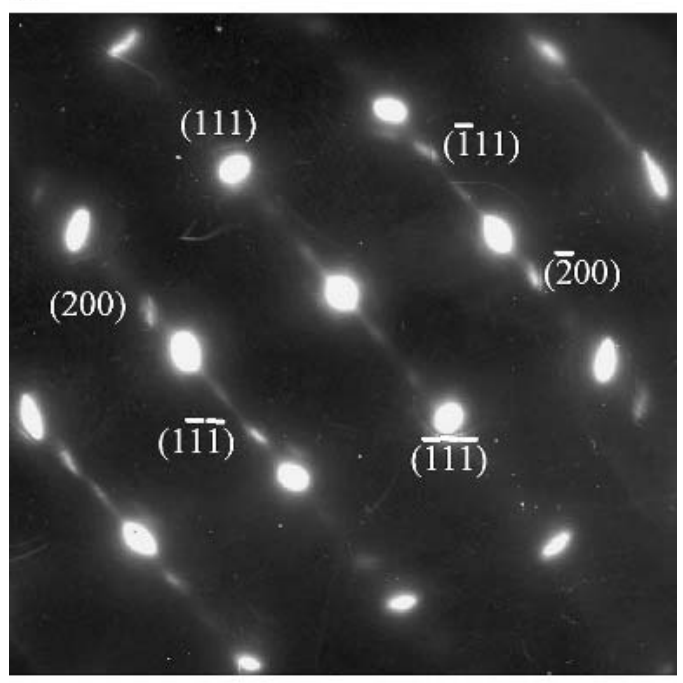

(b)

FIG. 2. A typical cross-sectional TEM image of (a) high-density mechanical microtwins and (b) the corresponding SAED pattern at a depth of about $22 \mu \mathrm{m}$.

across twin-matrix interfaces was found to be up to several degrees.

The deviation in the orientation angles of the microtwins from that of regular twins with a mirror lattice symmetry suggests the existence of a high density of defects inside the microtwins and/or at the twin-matrix interfaces. This is confirmed by HRTEM observations of the microtwins, as shown in Fig. 3. In this atomic image of a microtwin, one can clearly recognize many dislocations that accumulate at the twin-matrix interface. A

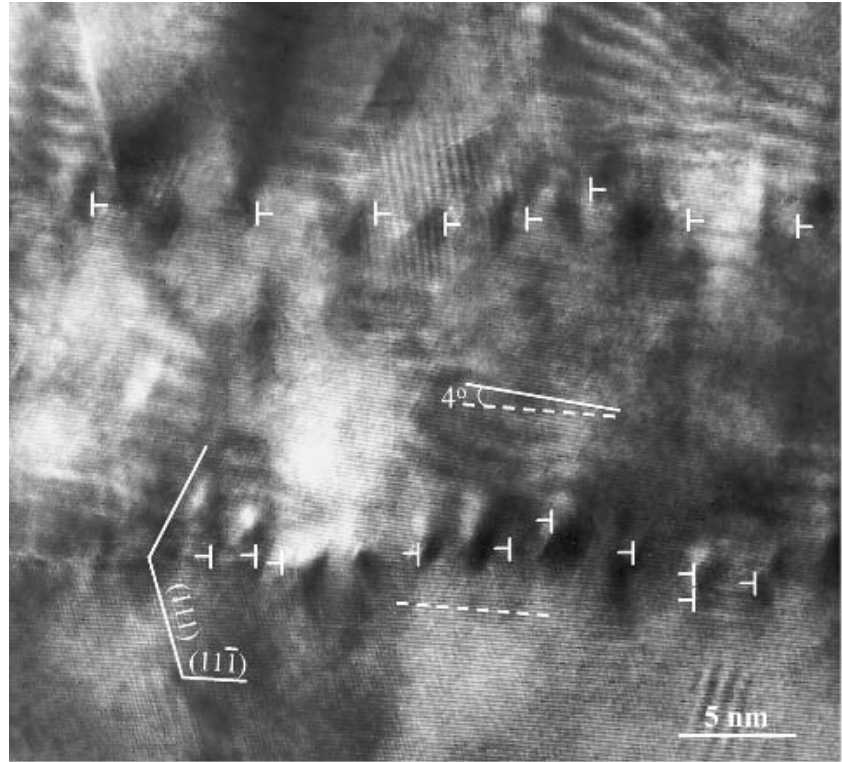

FIG. 3. HRTEM image of a microtwin taken along the [011] direction, showing the high density of dislocations at the twin boundaries and deviation in the orientation angle of the microtwins from that of regular twins with a mirror lattice symmetry.

prominent feature is that the dislocations located on the twin-matrix interface possess the same sign, which is the opposite of that of the dislocations on the neighboring twin-matrix interfaces. The Burgers vectors of these dislocations are perpendicular to the twin-matrix interfaces. The dislocation density was measured to be $\sim 2 \times$ $10^{17} \mathrm{~m}^{-2}$ for the twin-matrix interfaces on both sides of the microtwin. With such a high density of twin boundary dislocations for a nanometer-thick twin, a significant orientation deviation will be induced for the twin relative to the matrix. Estimating the deviation angle as the dislocation Burgers vector divided by the spacing of corresponding dislocations, one obtains a deviation of several degrees for the orientation angle across the twin-matrix interface, which is consistent with the measured value of approximately $4^{\circ}$, as illustrated in Fig. 3.

Close inspection of the mechanical microtwins revealed dislocations in the interior of the microtwins. Typical microstructural images of the mechanical microtwins are shown in Fig. 4. The evident contrast inside the microtwins and the matrix in Fig. 4(a) indicates a high level of microstrain. The dislocation density was estimated to be as high as $-3 \times 10^{17} \mathrm{~m}^{-2}$ inside the microtwins [Fig. 4 (b)]. To accommodate severe plastic deformation and to reduce the strain energy of the system, these dislocations should arrange themselves into low-energy dislocation configurations.

Figure 5 is a HRTEM image showing typical arrangements of dislocations inside a microtwin. It is seen that some dislocations form a dislocation array across the thickness of the microtwin, as indicated by (I) in Fig. 5. 


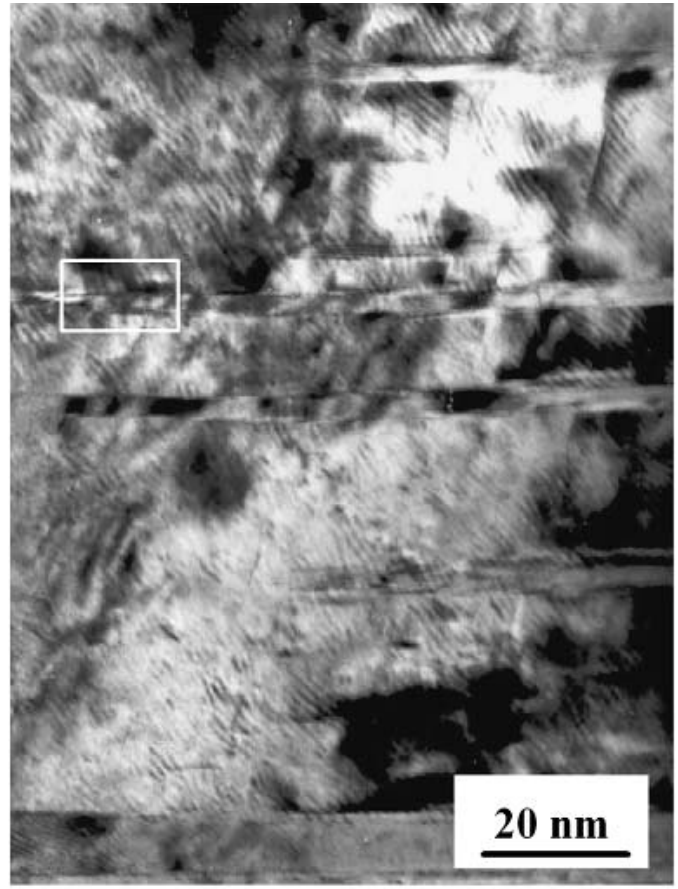

(a)

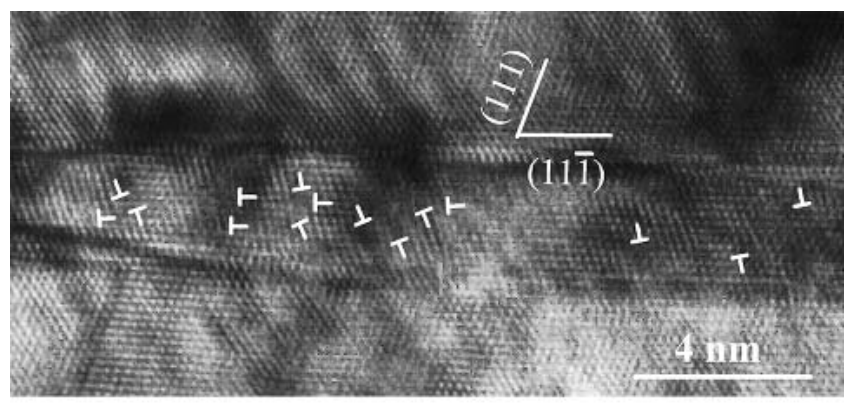

(b)

FIG. 4. (a) HRTEM image showing mechanical microtwins and matrix with severe plastic deformation; (b) magnified image of the region outlined in (a).

Another dislocation arrangement is characterized by aggregation of several dislocation pairs with opposite Burgers vectors forming dislocation multipoles in a very small region, labeled (II) in Fig. 5. These dislocations are arranged such that a tiny (nanometer-sized) block is surrounded by the dislocations, and an apparent misorientation exists across the dislocation multipoles. For example, the dislocation multipoles (II) in Fig. 5 induced a misorientation angle of about $16^{\circ}$ across the nanometersized block and the surrounding twin lattice. These two types of dislocation arrangements were frequently observed inside both the microtwin and the matrix lamellae. These dislocations are also responsible for the orientation deviations of the microtwins shown in Fig. 2.

During the SMAT processing with repeated multidirectional loading at high strain rates, more and more dislocations are generated inside both the microtwins and

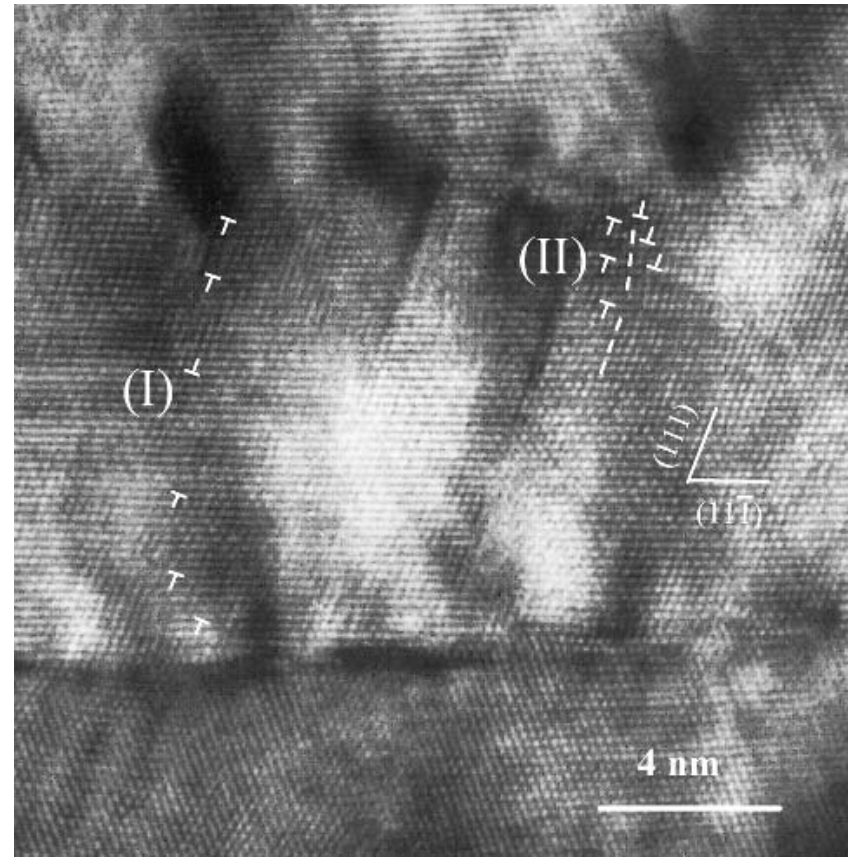

FIG. 5. HRTEM image of a microtwin taken along the [011] direction, showing typical dislocation arrangements: (I) dislocation arrays across the thickness of the microtwin and (II) dislocation multipole walls.

the matrix lamellae. When the dislocations approach each other within a certain distance, positive and negative edge dislocations traveling on different atomic slip planes in different directions may trap each other via the dipolar attraction and form dislocation dipoles. Frequent trapping events at larger strains cause the accumulation of positive and negative dislocations and eventually lead to dislocation multipole formation. A dislocation multipole possesses a relatively low energy state and does not generate a long-distance internal stress due to the equal number of positive and negative dislocations.

\section{Formation of nanometer-sized grains}

In the surface layer with an even larger strain, TEM observations showed that the orientation deviations for the mechanical microtwins became more pronounced. The diffraction spots in the SAED pattern are elongated to a greater degree, indicating a wider spread of orientations for both the microtwins and the nanometer-thick matrix lamellae, as shown in Fig. 6(b). Dark-field TEM images [as in Fig. 6(a)] showed discontinuous contrast along the microtwins (parallel bands), indicating that these lamellar structures are broken into equiaxed nanometer-sized blocks with different crystallographic orientations. Apparently, these nanometer-sized blocks originated from a continued increase in orientation deviation of nanometer-sized regimes surrounded by dislocation arrays, as in Fig. 5. With increasing strain, more dislocations are generated and form more (and/or denser) 


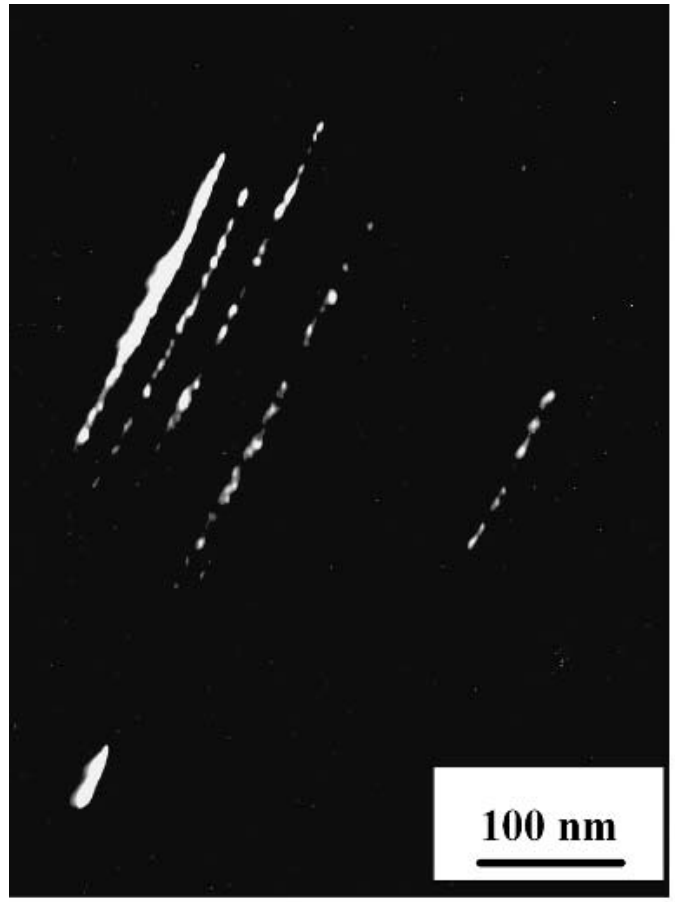

(a)

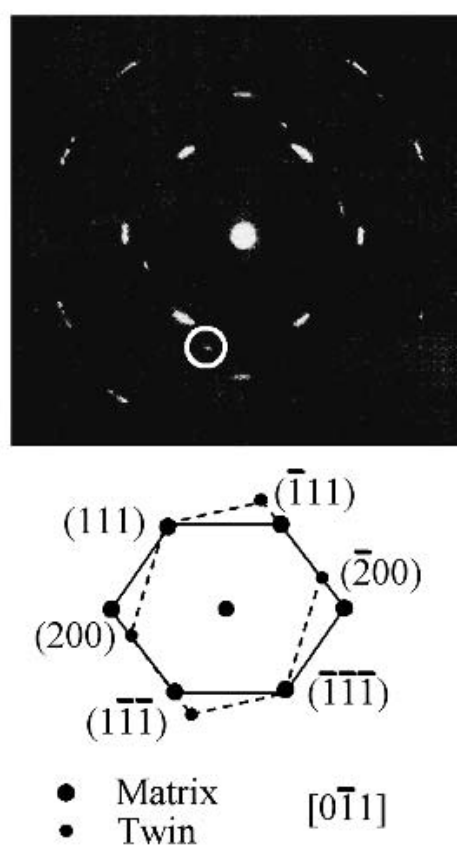

(b)

FIG. 6. (a) A cross-sectional dark-field TEM image taken from the SAED spot marked with a circle in (b), showing the evolution of mechanical microtwins into equiaxed nanometer-sized blocks.

dislocation arrays that gradually subdivide the microtwin lamellae into refined nanostructured blocks.

In the top surface layer with a much larger strain, the misorientations among the refined blocks increase continuously, eventually leading to the formation of nanometer-sized grains with random crystallographic orientations. Figure 7 is a typical TEM image showing the evolution of mechanical microtwins into randomly orientated nanocrystallites. The nanocrystallites are roughly equiaxed and exhibit random crystallographic orientations, as indicated by the continuous diffraction rings in the corresponding SAED pattern [Fig. 7(c)]. From the bright- and dark-field images, it is interesting to note that the nanocrystallites seem to be arranged in parallel bands. It is further noted that these adjacent parallel bands possess imperfect fcc twin relations, which were revealed by micro-area electron-diffraction patterns, as seen in Fig. 7(d). The deviation of twin diffraction spots from the regular positions for fcc twins is due to the change in orientation angle across matrix and microtwin. The experimental results described above clearly indicate that randomly orientated nanocrystallites in the top surface layer are derived from the nanometer-sized blocks within twin-matrix lamellae via formation of large-angle grain boundaries across these blocks. The interactions of mechanical microtwins with dislocations dominate the grain subdivision process.

In terms of the experimental observations and analysis, the formation mechanism of nanocrystallites in the surface of Inconel 600 by means of SMAT can be summarized as follows: (i) Formation of high-density mechanical microtwins divides the initial coarse grains into nanometer-thick twin-matrix lamellae; (ii) Formation of dislocation arrays further subdivides the twin-matrix lamellae into equiaxed nanometer-sized blocks; (iii) These nanometer-sized blocks evolve into randomly orientated nanocrystallites.

The grain refinement mechanism of coarse-grain polycrystalline materials during plastic deformation depends strongly on the crystal structure of the material and the extrinsic deformation conditions. Plastic deformation of metallic materials can be accommodated by slip and twinning. Generally, slip dominates the deformation process in materials with medium to high stacking fault energy (SFE), and twinning is favorable for low SFE metals, especially at high strain rates and/or low deformation temperatures. Therefore, for most metals and alloys with high SFE, such as $\mathrm{Ni}\left(128 \mathrm{~mJ} / \mathrm{m}^{2}\right)$ and $\mathrm{Al}$ $\left(166 \mathrm{~mJ} / \mathrm{m}^{2}\right),{ }^{20}$ strain-induced grain refinement processes are dominated by dislocation activity. ${ }^{12-16}$ In the current study of Inconel 600, which possesses a lowmedium SFE, ${ }^{21}$ the SMAT process may provide preferable conditions for development of microtwins due to the low deformation temperature (room temperature) and high strain rates that could suppress thermally activated dislocation processes. Instead, different refinement 


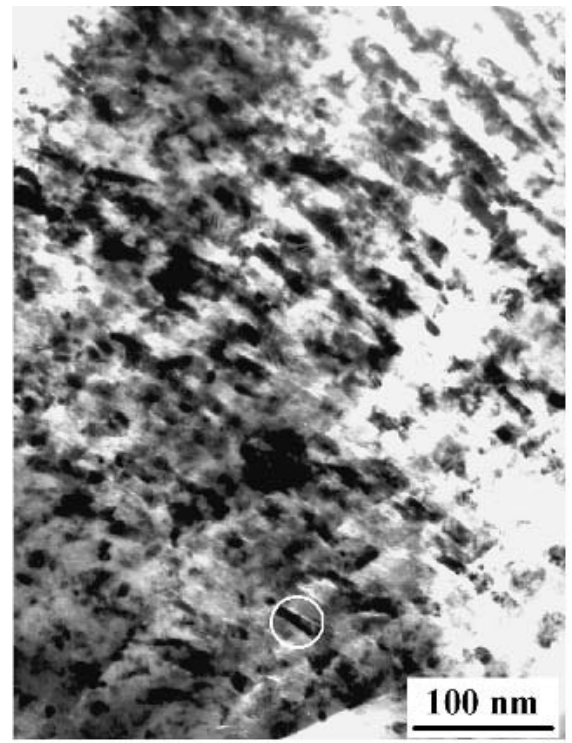

(a)

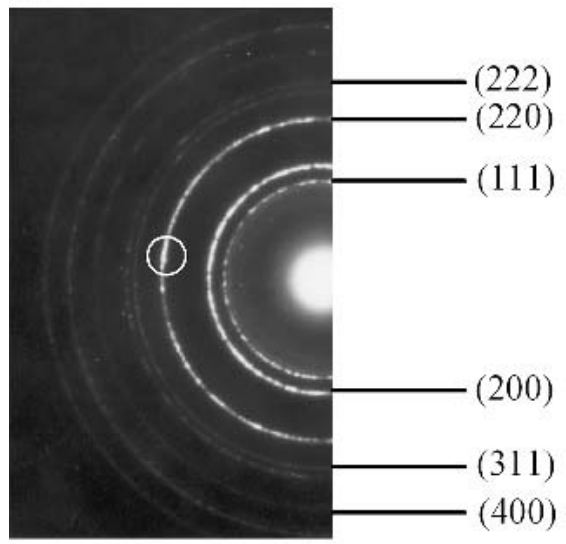

(c)

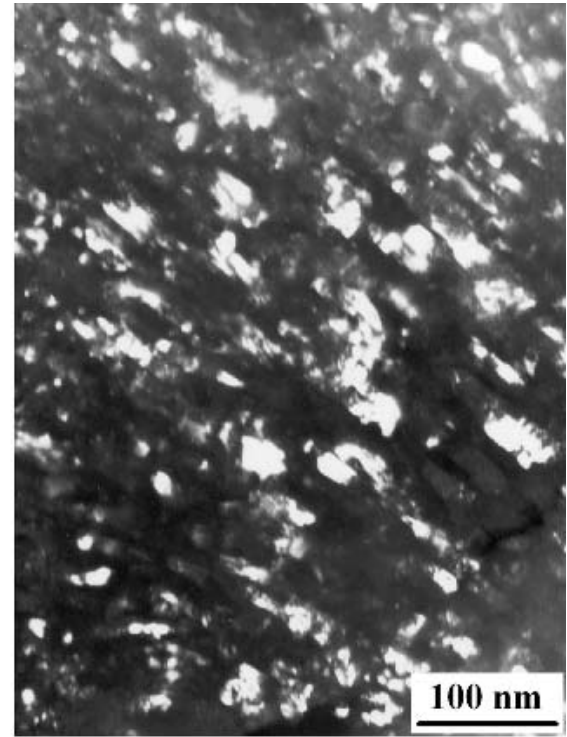

(b)

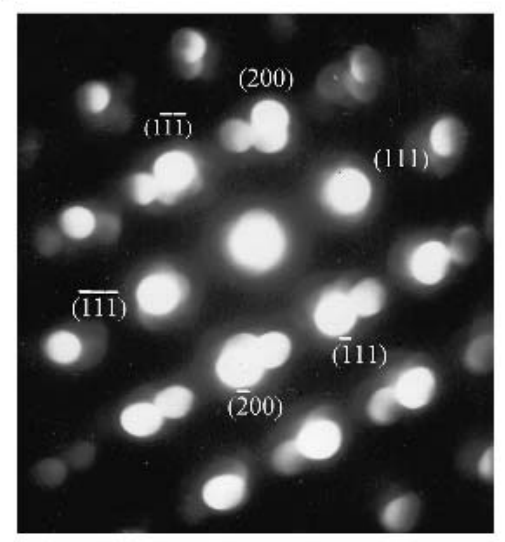

(d)

FIG. 7. (a) Bright-field and (b) dark-field TEM images taken from the SAED spot marked with a circle in (c) for the top surface layer of the SMAT sample; (c) the corresponding SAED and (d) the micro-area electron-diffraction patterns of the region outlined with a circle in (a).

mechanism is observed; that is, nanocrystallites are formed via formation of mechanical microtwins and the interaction of the microtwins with dislocations.

The experimental results showed that the spacing between adjacent mechanical microtwins decreases dramatically with increasing strain and strain rate in the surface layer. Within $15 \mu \mathrm{m}$ of the top surface, the thickness of the twin-matrix lamellae tends to a saturated value of about $8 \mathrm{~nm}$. No further reduction of the thickness is found with further increases in strain closer to the surface. This might be attributed to the saturated strain rate (and strain) achieved in the top surface layer due to the fixed SMAT processing parameters. Formation of mechanical microtwins (and stacking faults) within such thin lamellae seems to be inhibited, due to the effective confinement of the twin boundaries. Further straining of the thin twin-matrix lamellae is accommodated by formation of dislocations that arrange themselves into dislocation arrays and multipoles, rather than forming additional microtwins. Those dislocation arrangements act as precursors for the formation of more grain boundaries that refine the nanometer-thick lamellae into equiaxed nanograins. Thus, the grain refinement mechanism is also dependent on the dimension (or confinement) of grains to be refined. For Inconel 600 under the same deformation conditions, mechanical twinning dominates the refinement of coarse grains, but dislocation activity refines the nanometer-thick lamellae into equiaxed nanograins when formation of mechanical microtwins becomes too difficult.

\section{SUMMARY}

The microstructure of the surface layer of an Inconel 600 plate was refined into equiaxed, randomly oriented nanocrystallites by means of SMAT at room temperature. 
Cross-sectional TEM and HRTEM observations revealed that the strain-induced grain refinement process includes (i) formation of a high density of mechanical microtwins that subdivide the original coarse grains into twin-matrix lamellae, (ii) development of dislocation arrays further subdivides the twin-matrix lamellae into equiaxed nanometer-sized blocks, and (iii) these nanometer-sized blocks evolve into randomly oriented nanocrystallites. Such a grain refinement process involving the interaction of microtwins and dislocations, which differs from those observed in other materials in the literature, indicates that the strain-induced grain refinement mechanism is sensitive to the crystal structure of the material and the deformation conditions. In addition, the experimental results in the current study clearly show that the plastic deformation (or the strain-induced refinement) mechanism of crystallites is also dependent on their dimensions.

\section{ACKNOWLEDGMENTS}

Financial support from the National Science Foundation of China, the Ministry of Science and Technology of China (Grant G1999064505), Framatone Corporation (France), and the Ministry of Research of France (Grant 2001882, CPER EN2040) is acknowledged. The authors would like to acknowledge Professor Z.G. Wang and S.X. Li for fruitful discussions. One of the authors (N.R. Tao) also gratefully acknowledges Dr. T.J. Balk for critical reading of the manuscript.

\section{REFERENCES}

1. C.C. Koch: The synthesis and structure of nanocrystalline materials produced by mechanical attrition. Nanostruct. Mater. 2, 109 (1993).

2. H.J. Fecht, in Nanophase Materials, edited by G.C. Hadjipanayis and R.W. Siegel (Kluwer Academic Publishers, Dordrecht, The Netherlands, 1994), p. 125.

3. N. Hansen: Cold deformation microstructures. Mater. Sci. Tech. 6, 1039 (1990)

4. Y.M. Wang, M.W. Chen, H.W. Sheng, and E. Ma: Nanocrystalline grain structures developed in commercial purity $\mathrm{Cu}$ by lowtemperature cold rolling. J. Mater. Res. 17, 3004 (2002).
5. R.Z. Valiev, R.R. Mulyukov, V.V. Ovchinnikov, and V.A. Shabashov: Mössbauer analysis of submicrometer grained iron. Scr. Metall. Mater. 25, 2717 (1991).

6. Y. Iwahashi, Z. Horita, M. Nemoto, and T.G. Langdon: The process of grain refinement in equal-channel angular pressing. Acta Mater. 46, 3317 (1998).

7. A.P. Zhilyaev, S. Lee, G.V. Nurislamova, R.Z. Valiev, and T.G. Langdon: Microhardness and microstructural evolution in pure nickel during high-pressure torsion. Scr. Mater. 44, 2753 (2001).

8. R.Z. Valiev and I.V. Alexandrov: Nanostructured materials from severe plastic deformation. Nanostruct. Mater. 12, 35 (1999).

9. N.R. Tao, M.L. Sui, J. Lu, and K. Lu: Surface nanocrystallization of iron induced by ultrasonic shot peening. Nanostruct. Mater. 11, 433 (1999).

10. W.P. Tong, N.R. Tao, Z.B. Wang, J. Lu, and K. Lu: Nitriding iron at lower temperatures. Science 299, 686 (2003).

11. N.R. Tao, Z.B. Wang, W.P. Tong, M.L. Sui, J. Lu, and K. Lu: An investigation of surface nanocrystallization mechanism in $\mathrm{Fe}$ induced by surface mechanical attrition treatment. Acta Mater. 50, 4603 (2002).

12. A. Belyakov, T. Sakai, H. Miura, and K. Tsuzaki: Grain refinement in copper under large strain deformation. Philos. Mag. A 81, 2629 (2001).

13. D.A. Hughes: Scaling of deformation-induced microstructures in fcc metals. Scr. Mater. 47, 697 (2002).

14. D.A. Hughes and N. Hansen: Microstructure and strength of nickel at large strains. Acta Metall. 48, 2985 (2000).

15. B. Bay, N. Hansen, D.A. Hughes, and D. Kuhlmann-Wilsdorf: Evolution of f.c.c. deformation structures in polyslip. Acta Metall. 40, 205 (1992).

16. X. Wu, N. Tao, Y. Hong, B. Xu, J. Lu, and K. Lu: Microstructure and evolution of mechanically-induced ultrafine grain in surface layer of Al-alloy subjected to USSP. Acta Mater. 50, 2075 (2002).

17. D.H. Shin, I. Kim, J. Kim, and K.T. Park: Grain refinement mechanism during equal-channel angular pressing of a lowcarbon steel. Acta Mater. 49, 1285 (2001).

18. K. Lu, J. Lu, Chinese Patent No. 01122980. 2 (2001); French Patent No. FR2812284 (2001).

19. J.M. Manero, F.J. Gil, and J.A. Planell: Deformation mechanisms of Ti-6Al-4V alloy with a martensitic microstructure subjected to oligocyclic fatigue. Acta Mater. 48, 3353 (2000).

20. L.E. Murr, in Interfacial Phenomena in Metals and Alloys (Techbooks, Herndan, VA, 1975), p. 145.

21. M. Kumar, A.J. Schwartz, and W.E. King: Microstructural evolution during grain boundary engineering of low to medium stacking fault energy fcc materials. Acta Mater. 50, 2599 (2002). 